\title{
Estudio comparado sobre preferencias profesionales de doctorados en ingeniería. Colombia-Estados Unidos
}

\section{Jorge Enrique Celis-Giraldo}

Universidad Nacional de Colombia, Colombia

jecelisg@unal.edu.co

\section{Mauricio Duque-Escobar}

Universidad de los Andes, Colombia maduque@uniandes.edu.co

\section{Resumen}

A pesar de que se esperaría que la mayoría de los graduados de programas de doctorado en ingeniería laboren en organizaciones no académicas, como la industria, el mercado de trabajo muestra que la educación superior es el principal sector de empleo para un gran número de ellos. Para comprender este fenómeno se realizó un estudio comparado sobre las preferencias profesionales de los estudiantes de doctorado en ingenieria en Colombia y Estados Unidos. Los resultados de la comparación entre las preferencias profesionales muestran que los estudiantes optan por una carrera académica porque encuentran que ella les permite publicar, recibir reconocimiento de pares, libertad para escoger sus proyectos de investigación y estabilidad laboral; mientras que aquellos interesados en la industria muestran una preocupación por los salarios, fondos, recursos y acceso a equipos y tecnologías de punta.

\section{Palabras clave}

Doctorados en ingeniería, educación superior, educación comparada, mercado laboral, Colombia-Estados Unidos. (Fuente: Tesauro de la Unesco).

Recepción: 2013-11-05 / Envío a pares: 2014-04-16 / Aceptación por pares: 2014-04-29 / Aprobación: 2014-07-17 DOI: 10.5294/edu.2014.17.2.6

Para citar este artículo / To reference this article / Para citar este artigo

Celis-Giraldo, J. E. y Duque-Escobar, M. (2014). Estudio comparado sobre preferencias profesionales de doctorados en ingeniería. Colombia-Estados Unidos. Educ. Educ. 17 (2), 306-320. Doi. 10.5294/edu.2014.17.2.6 


\title{
A Comparative Study of Professional Preferences Among Engineering Ph.D. Colombia-USA
}

\begin{abstract}
Although we expect most graduates of doctoral programs in engineering to find work with non-academic organizations, such as those in industry, the job market shows the higher education sector employs a large number of them. A study comparing the occupational preferences of engineering Ph.D. candidates in Colombia and the USA was conducted to understand this phenomenon. The results of that comparison of professional preferences shows students choose an academic career because they find it allows them to publish, to receive peer recognition, to have the freedom to choose their research projects, and provides job security; while those interested in industry show concern over wages, money, resources and access to high-end equipment and technology.
\end{abstract}

\section{Key words}

Ph.D. in engineering, higher education, comparative education, job market, Colombia-USA. (Source: Unesco Thesaurus). 


\section{Estudo comparado sobre preferências profissionais de doutorados em engenharia. Colômbia-EUA}

\section{Resumo}

Embora se espere que a maioria dos formados em programas de doutorado em engenharia trabalhe em organizações não acadêmicas, como a indústria, o mercado de trabalho mostra que a educação superior é o principal setor de emprego para um grande número deles. Para compreender esse fenômeno, realizou-se um estudo comparado sobre as preferências profissionais dos estudantes de doutorado em engenharia na Colômbia e nos Estados Unidos. Os resultados da comparação entre as preferências profissionais mostram que os estudantes optam pela carreira acadêmica porque constatam que ela lhes permite publicar, receber reconhecimento de pares, liberdade para escolher seus projetos de pesquisa e estabilidade laboral; enquanto aqueles interessados na indústria mostram uma preocupação pelos salários, fundos, recursos e acesso a equipamentos e tecnologias de ponta.

\section{Palavras-chave}

Doutorados em engenharia, educação superior, educação comparada, mercado laboral, Colômbia-EUA. (Fonte: Tesauro da Unesco). 


\section{Introducción}

El fortalecimiento de las capacidades de innovación del sector industrial ha sido definido como uno de los factores más importantes para estimular el crecimiento económico del cual depende en gran parte el mejoramiento de la calidad de vida de los ciudadanos en los diferentes países (WB, 2010). El incremento de profesionales con título de doctorado en la industria es una condición básica para promover innovación basada en conocimiento (Lee, Miozz y Laredo, 2010) que tenga como resultado una novedad, mayor o menor, sobre los procesos, los productos, la organización o la comercialización (OECD, 2009). Los graduados de doctorado por lo general han recibido una educación enfocada en la generación, el uso y la difusión de conocimiento (OECD, 2010) y, por esta razón, son vistos como aquel recurso humano con capacidad de transferir y aplicar conocimiento de punta a la industria, aspecto fundamental en la capacidad de absorción de este sector que hace posible la producción de innovación (Herrera, Muñoz y Nieto, 2010). Los graduados de doctorado en ingeniería contribuyen especialmente a elevar los niveles de productividad en comparación con graduados de otras áreas de conocimiento (Casey, 2009).

A pesar de que en ingeniería se esperaría que la mayoría de los graduados de doctorado formados en la universidad laboren en organizaciones no académicas, el mercado de trabajo en ingeniería muestra que la educación superior es el principal sector de empleo para un gran número de doctores que trabajan como investigadores. Por ejemplo, en países como España, Portugal, Rumania, Turquía y Colombia, algo más del $80 \%$ de los graduados de doctorado en ingeniería trabajan en el sector de la educación, y en países como Polonia este porcentaje es igual al 98 \% (Auriol, Misu y Freeman, 2013; Universidad del Rosario, 2010). Aunque estas cifras contrastan con aquellas de países como Estados Unidos, Holanda, Bélgica y Noruega, en los cuales la educación superior emplea entre el 30 y el 56\%, hay más graduados de doctorado en ingeniería trabajando en educación superior que en el gobierno y en el sector sin ánimo de lucro (Auriol et al., 2013).

Una posible explicación a este fuerte interés en trabajar en la educación superior sería que los salarios en dicho sector son más altos que en los demás sectores, asumiendo que este es el principal factor sobre el cual los doctores basan su decisión de trabajar en cierto sector de la economía y no en otro. Pero la evidencia muestra que aquellos graduados de doctorado que trabajan como investigadores "son mejor pagados en el sector externo a la academia en muchos países" (Auriol et al., 2013, p. 27). Lo paradójico es que esta diferencia entre los ingresos según sector es reconocida por los mismos graduados de doctorado. Al observar los niveles de satisfacción que ellos tienen sobre sus actuales empleos (ubicación, grado de independencia, retos intelectuales, grado de responsabilidad, contribución a la sociedad, estabilidad laboral, salario, condiciones laborales, estatus social, oportunidades para desarrollarse profesionalmente, beneficios), se encuentra que los graduados de doctorado no están satisfechos con sus salarios ni con sus beneficios. A pesar de esta falta de satisfacción, los doctores tienen una alta satisfacción con el grado de independencia y de responsabilidad (Auriol et al., 2013). Lo anterior parece sugerir que los beneficios no monetarios, tales como la independencia y responsabilidad percibidas por los graduados de doctorado, son considerados unos incentivos significativos para optar por un empleo en la academia, a pesar de los salarios que obtienen en dicho sector (Casey, 2009).

En este contexto, la pregunta que surge es por qué los graduados de doctorado en ingeniería tienen una mayor inclinación a trabajar en la academia antes que en la industria, especialmente cuando su formación doctoral está aparentemente más vinculada con esta rama y sus habilidades para realizar investigación son vitales para la innovación del sector industrial. Al respecto, algunos estudios han encontrado que las preferencias profesionales 
de los estudiantes de doctorado en ingeniería (por ejemplo, retos intelectuales, posibilidades de escoger sus agendas de investigación y publicar) tienen una influencia importante en las decisiones que ellos toman sobre trabajar en la academia o en la industria (Roach y Sauermann, 2010; Thune, 2009). Al analizar las preferencias profesionales de los estudiantes es posible comprender por qué algunos tienen una fuerte inclinación por seguir una carrera académica, por un lado, y, por otro, la manera en que dichas preferencias contribuyen a explicar la configuración del mercado de los ingenieros con doctorado.

En este marco, el propósito de este artículo es comparar las preferencias profesionales de aquellos estudiantes de doctorado en ingeniería que aspiran a trabajar en la academia con la de aquellos que buscan un empleo en el sector industrial con base en los resultados obtenidos de dos encuestas similares aplicadas en Colombia y en Estados Unidos. Esta comparación no solo permite analizar las diferencias y las similitudes entre aquellos estudiantes interesados en alcanzar una carrera académica de aquellos que se encuentran en la industria, sino también las del mercado de doctores en ingeniería en Estados Unidos y Colombia que, como se mostrará más adelante, son similares a pesar de las diferencias en la edad de graduación, las tasas de empleabilidad y el número de doctores que trabajan en la industria.

El artículo está organizado como sigue: la primera sección señala la importancia que tienen para los estudios comparados en educación las preferencias profesionales especialmente cuando se trata de hacer comparaciones entre un país desarrollado y uno en desarrollo como es el caso de Estados Unidos y de Colombia. Posteriormente se hace una breve caracterización de los graduados de doctorado en general y los de ingeniería en particular (número, género, tasa de empleabilidad, porcentaje de doctores en la industria) en Estados Unidos y en Colombia para tener así una idea básica de algunas características del mercado de doctores. Luego se describe la metodología utilizada para hacer la comparación dando especial énfasis a las preferencias profesionales para comprender las dinámicas del mercado de trabajo de los doctores en ingeniería y se discuten los resultados de dicha comparación. Finalmente, se plantean algunos temas de investigación que podrían orientar estudios destinados a expandir la comprensión sobre otros factores que inciden en la configuración de las preferencias profesionales de los estudiantes de doctorado en ingeniería y que permitirían al mismo tiempo comprender la configuración del mercado de los doctores.

\section{Los estudios comparados en educación y el mercado de los doctores}

Los estudios comparados en educación pueden ser definidos como un campo interdisciplinario que, con la aplicación de técnicas de recolección y análisis variados busca presentar de manera inteligible y organizada las diferencias y similitudes entre fenómenos educativos ubicaciones en contextos social, política y económicamente distintos o con algunas similitudes; dichos contextos pueden ser de tipo regional (por ejemplo Asia o América Latina), nacional y local (regiones ubicadas en un mismo país) y comparar aspectos de la educación en particular y la sociedad en general (currículo, métodos de enseñanza, mercados laborales) de acuerdo con un grupo poblacional determinado (género, estudiantes de un nivel educativo determinado) (Bray, Adamson y Mason, 2007b; Epstein, 1994).

Dentro de los estudios comparados, uno de los temas que ha sido definido como significativo es el del mercado laboral (Bray, Adamson y Mason, 2007a). Las preguntas que han orientado la investigación en la materia han estado encaminadas a analizar los efectos que la educación tiene sobre el desarrollo de las sociedades y los ingresos monetarios de los individuos a lo largo del tiempo de acuerdo con el grado de escolaridad alcanzado y, finalmente, las demandas que el sector económico impone a la educación 
para que ella las satisfaga (Cowen, 2009a). Sin embargo, poca atención han recibido las preferencias profesionales que los actores sociales tienen sobre un sector determinado de empleo ante todo las que tienen los estudiantes previo a su ingreso al mercado laboral (Sauermann y Roach, 2012). A pesar de la relevancia que tienen la experiencia y la subjetividad en los estudios comparados (Fairbrother, 2007; Potts, 2007), aquellos sobre el mercado de trabajo de los doctores se han concentrado fundamentalmente en trazar las diferentes rutas que siguen los graduados de doctorado (edad de ingreso al mercado de trabajo, primer y segundo empleos, ingresos, estabilidad laboral, sectores de la economía predominantes, niveles de satisfacción con las condiciones laborales) y la movilidad de doctores del sector público al sector privado (Auriol, 2010). Estas aproximaciones suelen tomar en consideración de manera casi exclusiva las dinámicas de la oferta y la demanda en el mercado laboral de los doctores mientras pasan por alto las preferencias subjetivas que tienen los graduados de doctorado en relación con la selección de una carrera en la academia o en la industria. En otras palabras, la dimensión subjetiva al parecer no se considera como un factor explicativo de la configuración del mercado de trabajo sino que las explicaciones sobre las elecciones hechas por los actores sociales - en este caso los estudiantes de doctorado- se entienden desde la disponibilidad de empleos en ciertos sectores de la economía. Algunos estudios señalan al respecto, que a pesar de que en países como Estados Unidos, en donde las plazas de profesores de tiempo completo son cada vez más escasas, los estudiantes siguen mostrando una inclinación importante por las carreras académicas lo cual pone en duda que los graduados de doctorado basen sus decisiones en el comportamiento del mercado laboral, y más bien lo hagan sobre las preferencias que tienen acerca de ciertas posiciones laborales en los sectores académico e industrial (Roach y Sauermann, 2010; Sauermann y Roach, 2012).

Por otra parte, la investigación disponible sobre los estudios comparados en mercados de tra- bajo de los doctores se ha hecho en su mayoría sobre países en Europa y desarrollados (Auriol, 2007, 2010; Auriol, Bernad y Schaaper, 2010; Auriol et al., 2013). Hay una escasez de estudios sobre países en América Latina y en desarrollo, excepto por el caso de Argentina (Auriol, 2007). Esta situación puede afectar la comprensión de las dinámicas del mercado laboral de los doctores en estos países que de manera sostenida en las dos últimas décadas han venido incrementando el número de graduados de doctorado por año (Jaramillo, 2010; Schwartzman, 2007), e igualmente, el poder identificar las diferencias y similitudes que puedan existir con países desarrollados.

Este artículo puede ser considerado como un aporte a los estudios comparados en educación dedicados a estudiar los mercados laborales de los doctores en ingeniería. Por una parte, hace un análisis del mercado laboral de los doctores desde las preferencias profesionales de los estudiantes de doctorado, una aproximación diferente de las que han prevalecido en los estudios comparados. Se busca estudiar el mercado laboral desde lo que los estudiantes de doctorado prefieren como destino laboral dando así cabida a la dimensión subjetiva como un aspecto significativo del mercado de trabajo. Por otra parte, este artículo provee conocimiento sobre el caso de Colombia como una manera de hacer comparaciones entre un país desarrollado y uno en desarrollo acerca de un fenómeno tan importante como lo es el mercado laboral de los doctores en ingeniería. Finalmente, puede contribuir a la definición de políticas públicas que teniendo en cuenta las preferencias profesionales de los estudiantes de doctorado busquen estimularlos a optar por una carrera en la industria. Entender las preferencias profesionales es un insumo básico para políticas que comprendan lo que los individuos perciben como el destino laboral deseable según sus expectativas subjetivas. 


\section{Algunas características de los graduados de doctorado en Estados Unidos y en Colombia}

Aunque Colombia ha incrementado el número de graduados de doctorado por cada $100 \mathrm{mil} \mathrm{ha-}$ bitantes - pasó de graduar 0,08 doctores en 2002 a 0,57 en 2011-, el número de nuevos graduados por año en Estados Unidos es evidentemente superior al de Colombia (figura 1). En 2011, por ejemplo, mientras Colombia graduó 0,57 nuevos graduados de doctorado, este número fue de 15,80 para el caso de Estados Unidos.

Figura 1. Graduados de doctorado por 100 mil habitantes en Estados Unidos y Colombia: 2002-2011

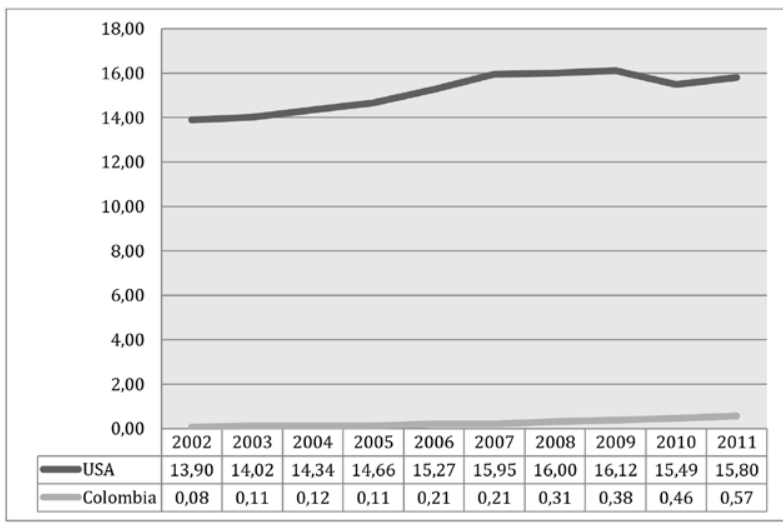

Fuente: NSF (2012b), OCYT (2012).

Esta pronunciada diferencia se puede explicar por el hecho de que la educación doctoral en Estados Unidos inició a finales del siglo XIX (Clark, 1997) mientras en Colombia comenzó al principio de los años ochenta (Lucio y Serrano, 1992). Para poner esta diferencia histórica en cifras, en 1900 las universidades en Estados Unidos otorgaron 150 títulos de doctorado (Thurgood, Golladay y Hill, 2006) y en Colombia las universidades alcanzaron los 173 títulos en 2009 (OCYT, 2012).

Por otra parte, al observar el porcentaje de nuevos estudiantes graduados en ingeniería con respecto al total de los nuevos graduados de docto- rado en todas las áreas del conocimiento entre los años 2002 y 2009, Colombia desde el 2004 ha otorgado más títulos de doctorado en ingeniería que los otorgados por Estados Unidos (figura 2). En otras palabras, en Colombia dos de cada diez nuevos graduados de doctorado eran doctores en ingeniería en 2009, y en Estados Unidos esta relación fue de uno de cada diez nuevos graduados.

Figura 2. Porcentaje de graduados

\section{de doctorado en ingeniería sobre el total de graduados de doctorado en Estados Unidos y Colombia: 2002-2009}

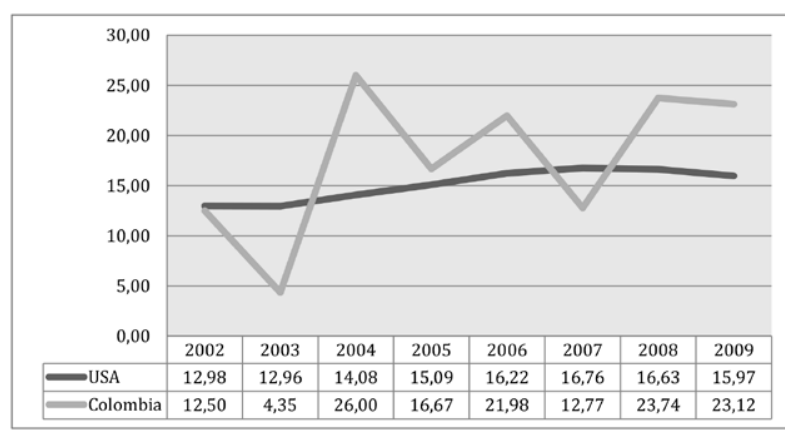

Fuente: NSF (2012a, 2012b), OCYT (2012)

Por otra parte, el número de nuevos estudiantes graduados en ingeniería por cada 100 mil habitantes se ha incrementado tanto en Estados Unidos como en Colombia entre los años 2002 y 2009 (figura 3). El número de graduados en ingeniería pasó de 0,01 en 2002 a 0,09 en 2009 en Colombia mientras en Estados Unidos el número creció de 1,80 graduados a 2,57 en el mismo periodo.

No obstante, el número de nuevos graduados en ingeniería por cada 100 mil habitantes en Colombia es inferior al de Estados Unidos. Para ponerlo en otras palabras, mientras en Colombia en 2009 se registraron 0,9 graduados, en Estados Unidos para el mismo año se registraron 2,57 graduados. Esto sugiere que las capacidades de innovación, en términos de recurso humano, son más fuertes en Estados Unidos que en Colombia. 


\section{Figura 3. Graduados de doctorado en ingeniería por $100 \mathrm{mil}$ habitantes en Estados Unidos y en Colombia: 2002-2009}

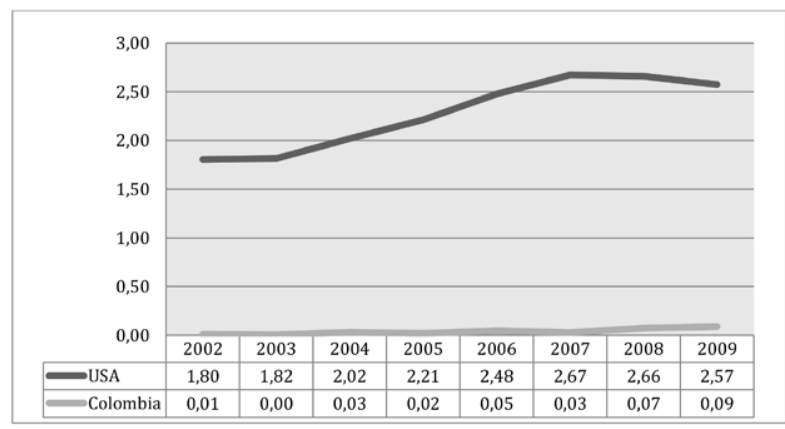

Fuente: NSF (2012a), OCYT (2012).

Entre los años 2002 y 2009, 20,1\% del total de los graduados de doctorado en ingeniería en Colombia fueron mujeres, y en Estados Unidos fueron del orden del 19,1\% (NSF, 2012a; OCYT, 2012). Por consiguiente, la ingeniería continúa siendo una disciplina predominantemente masculina.

Por otra parte, mientras la edad promedio de graduación de los estudiantes de doctorado en ingeniería en Estados Unidos es 31 años, en Colombia la edad promedio en que los estudiantes inician sus estudios de doctorado en ingeniería es 31 años (Auriol et al., 2013; Universidad del Rosario, 2010). Esto supone que los graduados de doctorado en Colombia son mayores que los graduados en Estados Unidos, y están ingresando al mercado de trabajo a una edad mayor con respecto a los graduados en ingeniería en Estados Unidos.

La información indica que las tasas de empleabilidad para los graduados de doctorado es más alta que aquellas exhibidas por otros graduados de la educación superior tanto en Estados Unidos como en Colombia. El 94\% de los graduados del doctorado en 2009 estaban en el sector formal de la economía en Colombia y el 80\% en 2008 para el caso de Estados Unidos (Auriol et al., 2013; OLE, 2010). La tasa de empleabilidad en Colombia es porcentualmente mayor a la que exhibe Estado Unidos; en 2008, la tasa de empleo en Estados Unidos para los hombres y las mujeres fue del 86 y el $84 \%$ respectivamente (Auriol et al., 2013). Aunque Colombia no provee diferencias de género en cuanto a las tasas de empleabilidad, la información señala que los hombres tienen ingresos superiores a los de las mujeres: los hombres ganan 300 mil pesos mensuales más que las mujeres al observar el salario del primer empleo.

Finalmente, en Estados Unidos el $6 \%$ del total de los trabajadores en ciencia y tecnología tenían título de doctorado en 2010 y en Colombia solamente el $0,06 \%$ de todos los trabajadores empleados tenían este título (NSF, 2012b; OCYT, 2012); el 35\% de todos los trabajadores con título de doctorado en Colombia pertenecían al área de la ingeniería, y $24 \%$ a la de ciencias sociales (OCYT, 2012).

\section{Metodología de comparación}

\section{¿Qué son las preferencias profesionales?}

Antes de describir las unidades de comparación que fueron seleccionadas para este artículo, es importante ofrecer una definición sucinta sobre las preferencias profesionales. La investigación en estudios comparados recomienda que antes de realizar una comparación es relevante hacer una aproximación conceptual para que esta tenga la confiabilidad y validez requeridas, máxime cuando se está comparando el mercado de los doctores en ingeniería en un país desarrollado como Estados Unidos y uno en desarrollo como Colombia a través de las preferencias profesionales (Bray et al., 2007b).

Las preferencias profesionales pueden ser definidas como el nivel de interés que tienen los estudiantes de doctorado en relación con un conjunto de atributos que están presentes en un empleo en la academia o en el sector empresarial. Los estudiantes de doctorado tienden a seleccionar aquellos empleos que están fuertemente relacionados con aquellos atributos laborales que ellos consideran están más alineados con su desarrollo profesional en el largo plazo. Roach y Sauermann señalan que 
"las preferencias de los estudiantes están fuertemente influenciadas por sus preferencias por varios atributos laborales" (2010, p. 432). En consecuencia, el proceso de decisión por un empleo depende más de las preferencias profesionales que tienen los estudiantes de doctorado; ellos orientan sus decisiones sobre la base de ciertos atributos cuando toman la decisión de obtener un empleo en la academia o en el sector industrial. Esta perspectiva, como se mencionó, supone que el mercado de trabajo no solo puede ser visto como una cuestión de oferta y demanda sino que también las preferencias juegan un papel preponderante a la hora de entender por qué los doctores en ingeniería se inclinan más por la academia que por la industria. La evidencia señala que definitivamente los salarios no tienen el mismo peso explicativo que las preferencias por la libertad y la responsabilidad, atributos que los graduados de doctorado suelen encontrar en la academia antes que en el sector industrial.

En el caso de los estudios comparados en educación esta aproximación resulta útil para contrastar un fenómeno como es el mercado de trabajo de los doctores en ingeniería en países tan disimiles como Colombia y Estados Unidos en términos de graduados de doctorado en ingeniería por año y los desarrollos relativos de cada uno de los sistemas de innovación.

Roach y Sauermann (2010) definieron diez atributos laborales que están presentes en las preferencias profesionales, a saber: salario y beneficios, disponibilidad de tecnologías de equipos y tecnologías de punta, disponibilidad de fondos y recursos, estabilidad laboral, responsabilidad, retos intelectuales, reconocimiento de pares, posibilidad de colaborar con otras instituciones/organizaciones, posibilidad de presentar y publicar resultados de investigación y libertad para escoger proyectos de investigación. Dichos atributos fueron seleccionados después de hacer análisis de la literatura y de discusiones con investigadores sobre aquellas cualidades que influyen en el proceso de decisión que siguen los estudiantes de doctorado cuando planean optar por un empleo en la industria o en la academia.

\section{Niveles de comparación}

En los estudios comparados en educación se encuentra que el cubo de Bray y Thomas es una herramienta metodológica útil para seleccionar los niveles de comparación (Bray et al., 2007a). El cubo incluye tres dimensiones relacionadas con el fenómeno por comparar: una primera relacionada con la ubicación geográfica y local (regiones del mundo, países, colegios, distritos, salones de clase, individuos), un segunda con grupos sin una ubicación geográfica específica (etnicidad, edad, religión, género, otros grupos, entre otros) y, por último, una con los aspectos de la educación y de la sociedad (currículo, métodos de enseñanza, financiación, mercado de trabajo, entre otros). De acuerdo con las comparaciones que se quieren realizar se pueden añadir nuevos elementos a las diferentes dimensiones y, a su vez, dichas comparaciones se pueden hacer en un tiempo determinado o comparando pasado con presente (Bray et al., 2007a, 2007b).

La tabla 1 presenta los niveles de comparación que fueron escogidos para contrastar las preferencias profesionales de los estudiantes a fin de analizar la configuración del mercado de los doctores en ingeniería en Estados Unidos y Colombia:

Tabla 1. Niveles de comparación

\begin{tabular}{|c|c|}
\hline $\begin{array}{c}\text { Nivel de comparación } \\
\text { pbicación geográfica: } \\
\text { países }\end{array}$ & $\begin{array}{c}\text { Estados Unidosy } \\
\text { Colombia }\end{array}$ \\
\hline $\begin{array}{c}\text { Grupos sin ubicación } \\
\text { geográfica }\end{array}$ & $\begin{array}{c}\text { Estudiantes de doctorado } \\
\text { en ingeniería }\end{array}$ \\
\hline $\begin{array}{c}\text { Aspecto de la educación y } \\
\text { la sociedad }\end{array}$ & $\begin{array}{c}\text { Mercado de trabajo de los } \\
\text { doctores en ingeniería }\end{array}$ \\
\hline
\end{tabular}

Fuente: adaptado de Bray et al. (2007a).

\section{Fuentes de información}

La información sobre las preferencias profesionales se obtuvo de dos estudios hechos en Estados 
Unidos y en Colombia (Celis, Duque y Ramírez, 2012; Roach y Sauermann, 2010). Ambos aplicaron una encuesta a estudiantes de doctorado en ingeniería para conocer sus preferencias profesionales sobre la academia y la industria. Mientras en Estados Unidos el estudio también incluyó estudiantes de ciencias básicas, en Colombia tuvo en cuenta profesores y graduados de doctorado en ingeniería. La encuesta contenía preguntas abiertas y cerradas (Roach y Sauermann, 2010). La tabla 2 contiene información básica sobre los encuestados:

Tabla 2. Información sobre los encuestados en Estados Unidos y en Colombia

\begin{tabular}{|c|c|c|}
\hline & Estados Unidos & Colombia \\
\hline $\begin{array}{c}\text { Número de } \\
\text { encuestados }\end{array}$ & $\begin{array}{c}476 \text { (tres } \\
\text { universidades) }\end{array}$ & $\begin{array}{c}82 \text { (cinco } \\
\text { universidades) }\end{array}$ \\
\hline Hombre & $46 \%$ & $87 \%$ \\
\hline Nacionalidad & $78 \%$ estadounidenses & $98 \%$ colombianos \\
\hline
\end{tabular}

Fuente: Celis et al. (2012), Roach y Sauermann (2010).

Es importante anotar que el porcentaje de mujeres que se encuentran realizando estudios de doctorado en ingeniería en Colombia es bastante bajo cuando se compara con el porcentaje de las que se encuentran en la misma condición en Estados Unidos. Además, al observar la nacionalidad de los encuestados, se puede aseverar que los programas de doctorado en Colombia, por lo menos los que corresponden al área de ingeniería, no son internacionales. Al contrario, están dedicados a formar estudiantes colombianos; parte de este fenómeno puede ser explicado si se tiene en cuenta la creación reciente de los programas de doctorado en Colombia que apenas tienen tres décadas de existencia.

La encuesta indagó sobre las preferencias profesionales por tres posiciones laborales en Investigación y Desarrollo, a saber: empresa establecida, empresa de reciente creación y profesor en una universidad. En primer lugar, la encuesta pidió a los encuestados calificar los atributos relacionados con las tres posiciones laborales. Se utilizó para ello una escala de 1 a 5 ( 1 = no atractivo, 5 = muy atractivo) a fin de medir el nivel de preferencia por las tres posiciones laborales. En cuanto a las preferencias profesionales para cada una de las posiciones los estudiantes debían valorar qué tan presentes estaban los diez atributos. Para este caso se empleó una escala de 1 a 3 ( 1 = bajo, 3 = alto) (Roach y Sauermann, 2010).

\section{Preferencias profesionales de los estudiantes de doctorado}

\section{Preferencias por tres posiciones laborales}

Mientras en Colombia los estudiantes están más interesados en trabajar en la academia que los estudiantes en Estados Unidos — quienes prefieren seguir una carrera en una empresa consolidada-. ambos grupos de estudiantes están menos interesados en trabajar en una empresa de reciente creación (figura 4).

\section{Figura 4. Nivel de preferencia por tres posiciones laborales}

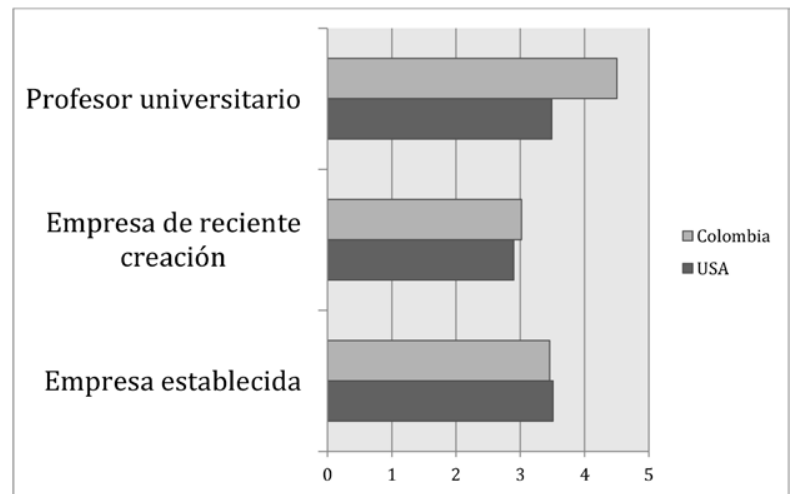

Fuente: Celis et al. (2012), Roach y Sauermann (2010).

El nivel de preferencia que tienen los estudiantes colombianos por la academia es "muy atractivo" $(4,5)$ comparado con la inclinación que tienen hacia una firma establecida o una de reciente creación. Por el contrario, los estudiantes estadounidenses tienen casi el mismo nivel de preferencia por un empleo en una empresa consolidada y en la academia. Este hecho parece sugerir que ellos consideran ambos sectores igual de atractivos para seguir una carrera a futuro. 
ISSN 0123-1294 | Educ.Educ. Vol. 17. No. 2 | Mayo-Agosto de 2014 | pp. 306-320.

Universidad de La Sabana | Facultad de Educación

\section{Preferencias por un empleo en una empresa consolidada}

El salario es el atributo laboral más atractivo para los estudiantes estadounidenses en una empresa consolidada. Sin embargo, para los colombianos la responsabilidad es el atributo más importante en este sector. Ambos estudiantes consideran que la libertad para escoger proyectos de investigación es el atributo más atractivo (figura 5). Atributos laborales usualmente asociados con la academia, tales como "posibilidad de colaborar con otras instituciones" y "reconocimiento de pares", son menos atractivos que atributos relacionados con "salario"y "equipos y tecnologías de punta".

\section{Figura 5. Preferencias por un empleo en una empresa establecida}

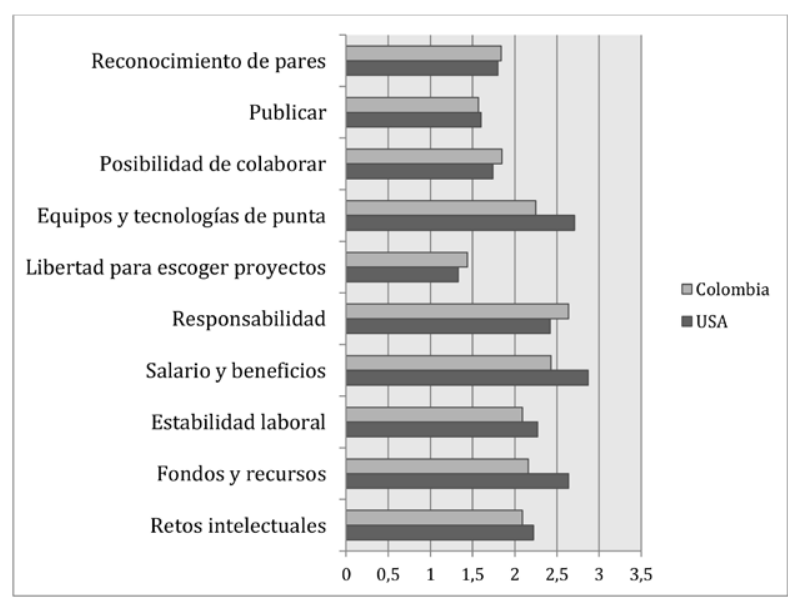

Fuente: Celis et al. (2012), Roach y Sauermann (2010).

Como se mostrará, los estudiantes norteamericanos reconocen que el salario en la academia es menos atractivo que el salario en las empresas constituidas y de reciente creación. En contrapartida, los estudiantes colombianos afirman que el salario es mucho más atractivo en la academia que en las empresas constituidas y de reciente creación (figuras 6 y 7). Mientras la percepción de los primeros parece estar más acorde con la situación del mercado laboral de los doctores en varios países en los cuales el sector privado ofrece salarios más altos que los de la academia (Auriol et al., 2013), la percepción de los estudiantes colombianos está en contradicción con esta evidencia.

\section{Preferencias por un empleo en una empresa de reciente creación}

Con respecto a una posición en una empresa de reciente creación, tanto los estudiantes norteamericanos como los colombianos están de acuerdo en que los fondos y recursos son los atributos laborales menos atractivos. Consideran, en cambio, que la responsabilidad, seguida por los retos intelectuales, son los atributos más atractivos en este tipo de empresas (figura 6).

\section{Figura 6. Preferencias por un empleo en una empresa de reciente creación}

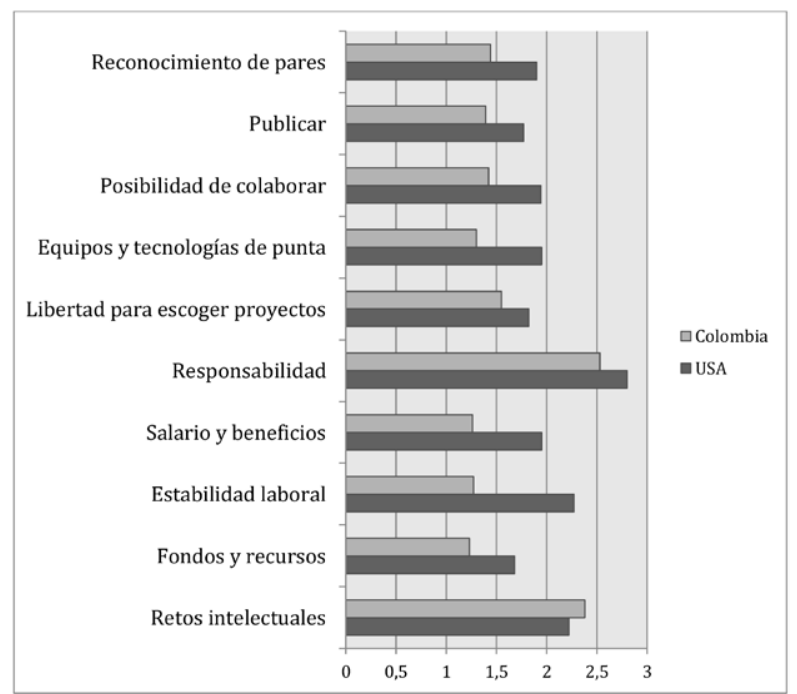

Fuente: Celis et al. (2012), Roach y Sauermann (2010).

El reconcomiendo de pares, publicar, la posibilidad de colaborar y la libertad para escoger proyectos son comúnmente encontrados en las empresas de reciente creación cuando se comparan las percepciones de los estudiantes norteamericanos con las de los estudiantes colombianos. Además, hay un notable contraste entre las percepciones de los estudiantes colombianos con las de los norteamericanos con respecto a la estabilidad laboral, pues los primeros piensan que este tipo de empresas no 
ofrece dicha estabilidad mientras los segundos afirman lo contrario.

\section{Preferencias por un empleo en la academia}

De acuerdo con ambos grupos de estudiantes, publicar es el atributo laboral más relevante en la academia. En contraste, mientras los estudiantes estadounidenses creen que el salario y los beneficios son los atributos menos atractivos, los colombianos valoran los fondos y recursos con la calificación menos baja (tabla 6). A pesar del hecho de que ambos grupos tengan la misma preferencia por el nivel de responsabilidad, los colombianos la perciben como esencial en la academia antes que en una empresa consolidada y una de reciente creación.

\section{Figura 7. Preferencias por un empleo en la academia}

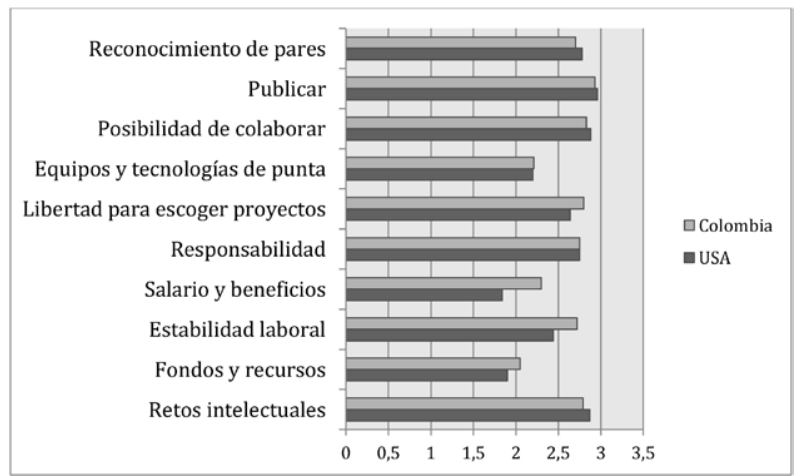

Fuente: Celis et al. (2012), Roach y Sauermann (2010).

A partir de la información recolectada es posible inferir que la academia es la posición laboral predilecta para los estudiantes colombianos pues ellos califican los diez atributos laborales con valores superiores a 2,5 (alto). Esto implica que los colombianos tienen una fuerte predilección por obtener una posición en la academia antes que en una empresa consolidada o de reciente creación. Este hallazgo ratifica el nivel de preferencia que tienen los estudiantes colombianos por la academia como se mostró en la figura 4. Además, es consistente con la percepción favorable que tienen los graduados de doctorado en ingeniería en Colombia sobre la posición de profesor universitario (figura 8).

Figura 8. Preferencias laborales de los graduados de doctorado en ingeniería por tres posiciones laborales

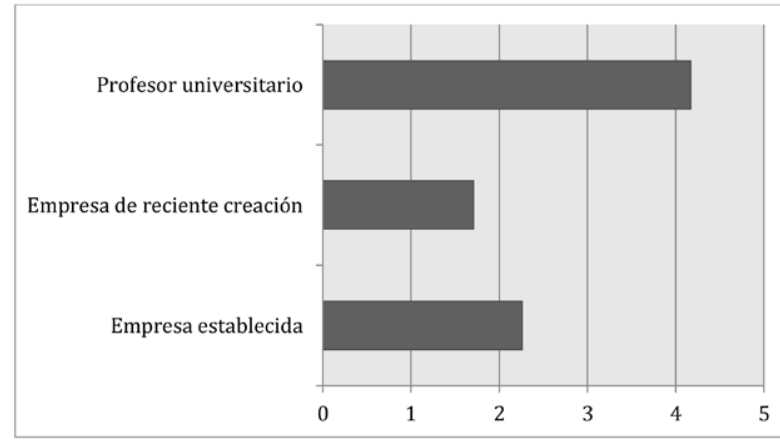

Fuente: Celis et al. (2012).

\section{Preferencias por un empleo en la academia $y$ en la industria}

Los estudiantes que aspiran a seguir una carrera académica tienden a mostrar una fuerte preferencia por la libertad para escoger proyectos, publicar, posibilidad para colaborar, reconocimiento de pares y estabilidad laboral. En contrapartida, aquellos que desean trabajar en el sector industrial valoran favorablemente los atributos relacionados con el salario, los fondos y recursos y el acceso a tecnología y equipos de punta (tabla 3).

\section{Tabla 3. Preferencias por un empleo en la academia o en el sector industrial}

\begin{tabular}{|c|c|}
\hline $\begin{array}{c}\text { Atributos laborales por la } \\
\text { academia }\end{array}$ & $\begin{array}{c}\text { Atributos laborales por el } \\
\text { sector industrial }\end{array}$ \\
\hline $\begin{array}{c}\text { Libertad para escoger } \\
\text { proyectos }\end{array}$ & Salario \\
\hline Publicar & Fondos y recursos \\
\hline Posibilidad para colaborar & $\begin{array}{c}\text { Tecnología y equipos de } \\
\text { punta }\end{array}$ \\
\hline Reconocimiento de pares & \\
\hline Estabilidad laboral & \\
\hline
\end{tabular}

Fuente: Celis et al. (2012), Roach y Sauermann (2010). 
Es evidente que los atributos mencionados por los estudiantes de doctorado que están interesados en una carrera en la universidad tienen una estrecha relación con los valores académicos, excepto por el caso de la estabilidad laboral. En cambio, las preferencias del otro grupo de estudiantes inclinados por la industria consideran atributos más vinculados con cuestiones monetarias y de capacidad de infraestructura.

\section{Conclusiones}

Este artículo mostró la importancia que tienen para los estudios comparados en educación las preferencias profesionales de los estudiantes de doctorado a fin de comprender la fuerte inclinación de algunos por seguir una carrera en la academia, y así poder explicar la manera en que se configura el mercado de los doctores en ingeniería en Estados Unidos y en Colombia. Por tal razón, las preferencias profesionales son una categoría conceptual también central para hacer comparaciones de los mercados de trabajo en ingeniería en países desarrollados y en desarrollo, pues se asume que estas tienen una incidencia en la configuración de los mercados de trabajo. La evidencia demostró que los graduados de doctorado no están satisfechos con sus salarios pero prefieren que sus posiciones laborales les permitan escoger sus temas de investigación, publicar y tener reconocimiento de pares. Este tipo de atributos laborales deben ser considerados a la hora de establecer políticas públicas encaminadas a incrementar el número de graduados de doctorado en ingeniería en el sector industrial.

Por otra parte, futuros estudios deben analizar si las preferencias profesionales cambian después de la inserción de los graduados de doctorado al mercado de laboral y, si fuera el caso, bajo qué circunstancias sucede. La experiencia laboral también tiene el potencial de afectar las preferencias profesionales en el tiempo como fue el caso del proceso de escolarización que tuvieron los estudiantes.

\section{Referencias}

Auriol, L. (2007). Labour Market Characteristics and International Mobility of Doctorate Holders: Results for Seven Countries. France: OECD.

Auriol, L. (2010). Careers of Doctorate Holders: Employment and Mobility Patterns. En OECD Science, Technology and Industry Working Papers. Paris: OCED.

Auriol, L., Bernad, F. \& Schaaper, M. (2010). Mapping Careers and Mobility of Doctorate Holders: Draft Guidelines, Model Questionare and Indicators. France: OECD.

Auriol, L., Misu, M. \& Freeman, R. (2013). Careers of Doctorate Holders: Analysis of Labour Market and Mobility Indicators. In OECD (ed.). OECD Science, Technology and Industry Working. Paris: OECD.

Bray, M., Adamson, B. \& Mason, M. (2007a). Different Models, Different Emphases Different Insights. In Bray, M., Adamson, B. \& Mason, M. (eds.). Comparative Education Research: Approaches and Methods (pp. 363-379). Hong Kong: Comparative Education Research Centre \& Springer. 
Bray, M., Adamson, B. \& Mason, M. (2007b). Introduction. In Bray, M., Adamson, B. \& Mason, M. (eds.). Comparative Education Research: Approaches and Methods (pp. 1-12). Hong Kong: Comparative Education Research Centre \& Springer.

Casey, B. (2009). The economic contribution of PhDs. Journal of Higher Education Policy and Managment, 31 (3), 219-227.

Celis, J., Duque, M. y Ramírez, C. (2012). Doctorados en ingeniería para promover la innovación: una propuesta para acrecentar la competitividad empresarial basada en la inserción de doctores en ingeniería en Colombia. Bogotá: ACOFI \& UniAndes.

Clark, B. R. (1997). Las universidades modernas: espacios de investigación y docencia. México: UNAM, Porrúa.

Cowen, R. (2009a). Industralisation, Knowledge Societies and Education. In Cowen, R. \& Kazamias, A. (eds.). International Handbook of Comparative Education (pp. 499-511). London: Springer Science.

Cowen, R. (2009b). Editorial Introduction: the National, the International, and the Global. In Cowen, R. \& Kazamias, A. (eds.). Reflections on the Development of Comparative Education (pp. 337-340). London: Springer Science.

Epstein, E. H. (1994). Comparative and International Education: Overview and Historical Development. In Torsten, H. \& Neville Postlethwaite, T. (eds.). The International Encyclopedia of Education (pp. 918-923). Oxford: Pergamon Press.

Fairbrother, G. P. (2007). Quantitative and Qualitative Approaches to Comparative Education. In Bray, M., Adamson, B. \& Mason, M. (eds.), Comparative Education Research: Approaches and Methods (pp. 39-62). Hong Kong: Comparative Education Research Centre \& Springer.

Herrera, L., Muñoz, M. \& Nieto, M. (2010). Mobility of public researches, scientific knowledge transfer, and the firm's innovation process. Journal of Business Research, 67, 510-518.

Jaramillo, H. (2010). La formación de posgrado en Colombia: maestrías y doctorados. Revista CTS, 13 (5), 131-155.

Kraemer-Mbula, E. \& Wamae, W. (2010). Innovation and the Development Agenda. Otawa: OECD, International Development Research Centre.

Lee, H., Miozz, M. \& Laredo, P. (2010). Career patterns and competences of PhDs in science and engineering in the knowledge economy: The case of graduates from a UK research-based university. Research Policy, 39, 869-891.

Lucio, R. y Serrano, M. (1992). Tendencias y politicas estatales. Bogotá: IEPRI- Universidad Nacional de Colombia.

NSF (2012a). Apendeix Table 2-27. Earned doctoral degrees, by citizenship, field, and sex: 2000-2009. In Science and engineering indicators 2012. USA: NSF.

NSF (2012b). Science and engineering labor force. In Science and engineering indicators 2012 (pp. 3-65). USA: NSF. 
ISSN 0123-1294 | Educ.Educ. Vol. 17. No. 2 | Mayo-Agosto de 2014 | pp. 306-320.

Universidad de La Sabana | Facultad de Educación

OLE (2010). Doctores graduados según sector de la economía en Colombia: 2001-2009. Colombia: Ministerio de Educación Nacional

OCYT (2012). Indicadores de ciencia y tecnología. Colombia 2012 [Science and Tecnology Indicators. Colombia 2012]. Bogotá: Observatorio Colombiano de Ciencia y Tecnología.

OECD (2009). Oslo Manual: Guidelines for collecting and interpreting innovation data. Paris: OECD.

OECD (2010). Measuring Innovation. New Perspective. Paris: OECD.

Potts, P. (2007). The Place of Experience in Comparative Education Research. In Bray, M., Adamson, B. \& Mason, M. (eds.). Comparative Education Research: Approaches and Methods (pp. 63-81). Hong Kong: Comparative Education Research Centre \& Springer.

Roach, M. y Sauermann, H. (2010). A taste for science? PhD scientists' academic orientation and self-selection into research careers in industry. Research Policy, 39, 422-434.

Sauermann, H. y Roach, M. (2012). Science PhD career preferences: levels, changes, and advisor encouragement. PLOSONE, 7 (5), e36307. doi:10.1371/journal.pone.0036307.

Schwartzman, S. (2007). Brazil's Leading University. Original Ideals and Contemporary Goals. In Philip, A. \& Balán, J. (eds.). Transforming Research Universities in Asia and Latin America World Calss Worldwide (pp. 143172). The United States of America:The Johns Hopkins University Press.

Thurgood, L., Golladay, M. J. \& Hill, S. T. (2006). U.S. Doctorates in the 2oth Century. Special Report. USA: National Science Foundation.

Thune, T. (2009). Doctoral students on the university-industry interface: a review of the literature. Higher Education, 58, 637-651.

Universidad del Rosario (2010). Evaluación del impacto del programa ACCES - Componente 2, apoyo a programas doctorales. Bogotá: Universidad del Rosario.

WB (2010). Innovation Policy. A Guide for Developing Countries. The United States of America: The World Bank. 